\title{
TWO SPACE-TIME INTERACTION TESTS FOR EPIDEMICITY*
}

\author{
BY \\ F. N. DAVID* AND D. E. BARTON \\ University of California, Berkeley, and University College, London
}

\section{INTRODUCTION}

Knox (1964), in discussing the pattern of incidence of childhood leukaemia in Northumberland and Durham, used a test for interaction in time and space which he had set out in an earlier paper (Knox, 1963). He was able only to conjecture the distribution of his test criterion. We have been able to show that his conjecture is substantially correct and that the distribution is Poisson in large samples. It is the purpose of this note first to give the exact mean and variance of Knox's criterion. If the distribution is assumed to be Poisson then the mean and variance should be approximately equal and in any practical problem the calculation of them both will serve as a guide to the adequacy of the assumption. Secondly, we give a space-time test of our own and apply it to Knox's data which he very kindly put at our disposal.

\section{Outline of Knox's Procedure}

A number of children have suffered from leukaemia. For each case the "date of onset" of the disease and the geographical co-ordinates of its domicile are recorded. Some clustering is noticeable in the observations if they are arranged in consecutive time order of date of onset. It is inevitable that there will be clustering in space if the domiciles are plotted on a map regardless of time order. It is desired to establish whether there is any association between the clustering in time and the clustering in space. Statistically we must test the assumption that there is no association so that time and space are independent of each other.

For the leukaemia data, Knox defines two observations as being adjacent in time if the interval between them is less than 60 days, and adjacent in space if the interval between them is less than $1 \mathrm{~km}$. Thus, for example, we have for the first ten time observations: the interval between the first and second observation is 30 , between the second and

* This investigation was supported (in part) by a research grant (No. G.M-10525) from the National Institutes of Health, United States Public Health Service. third 53, and so on, the series being $30,53,14,10$, $30,3,31,67,14$, and the total time between the first observation and the tenth is 252 days. We can plot these time-points. Since we are interested only in the adjacency of two points, we may plot them in arbitrary positions and join two points to denote adjacency; the actual distances we draw do not matter. Such a diagrammatic representation of a set of points, each pair either joined or not joined, is technically called a graph.

Thus, in the graph of these data (Figure) there are fifteen adjacencies or fifteen lines joining the points.
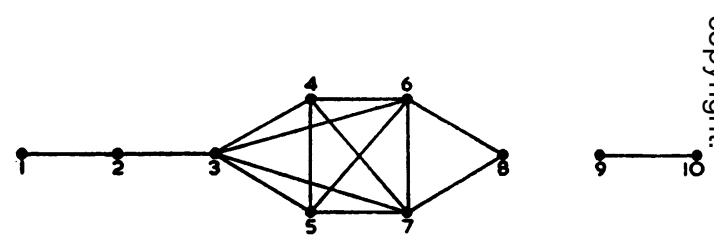

A similar diagram may be drawn for space.

If in the complete data $N_{1 S}$ denotes the number of adjacencies in space and $N_{1 T}$ the number of adjacencies in time then we may draw up a $2 \times 2$ table $\dagger$ as in Table I, where $X$ denotes the number of times that two points are adjacent in both time and space. The observations will not be independent because the same point will be used many times in determining the adjacencies. Thus $N$, the total number of

TABLE I

SCHEME OF KNOX'S $2 \times 2$ TABLE

\begin{tabular}{l|c|c|c}
\hline Time & Adjacent & Not Adjacent & Totals \\
\hline $\begin{array}{l}\text { Adjacent } \\
\text { Not adjacent }\end{array}$ & $\begin{array}{c}X \\
N_{1 S}-X\end{array}$ & $\begin{array}{c}N_{1 T}-X \\
N-N_{1 S} \\
-N_{1 T}+X\end{array}$ & $\begin{array}{c}N_{1 T} \\
N-N_{1 T}\end{array}$ \\
\hline Totals .. & $N_{1 S}$ & $N-N_{1 S}$ & $N$ \\
\hline
\end{tabular}

† Often called a four-fold table in medical contexts: see Hill (1961). 
ways in which the total number, $n$, of points may be joined in pairs is equal to $n(n-1) / 2$. Hence the standard analysis of $2 \times 2$ tables is not necessarily applicable. Taking 96 cases of childhood leukaemia (Knox, 1964, p. 21) obtains the data shown in Table II.

TABLE II

ALL POSSIBLE PAIRINGS OF 96 LEUKAEMIA CASES CLASSIFIED IN $2 \times 2$ TABLE BY MEANS OF TIME AND SPACE ADJACENCIES. FROM KNOX (1964)

\begin{tabular}{|c|c|c|c|}
\hline Time & Adjacent & Not Adjacent & Totals \\
\hline $\begin{array}{l}\text { Adjacent } \\
\text { Not adjacent }\end{array}$ & $\begin{array}{r}5 \\
20\end{array}$ & $\begin{array}{r}147 \\
4,388\end{array}$ & $\begin{array}{r}152 \\
4,408\end{array}$ \\
\hline Totals . & 25 & 4,535 & 4,560 \\
\hline
\end{tabular}

Under the assumption that $X$ is distributed as a Poisson variable, with parameter equal to $152 \times 25 / 4560=5 / 6$, the chance of obtaining five or more adjacencies in all possible permutations of the 96 points in time and space is 0.0017 . Thus we would (as Knox did) reject the null hypothesis and conclude there was evidence of interaction between time and space.

\section{Moments of $X$}

Using a graph-theoretic approach, we have been able to obtain an expression for the general $\mathrm{r}^{\text {th }}$ factorial moment of $X$ and consequently to establish under quite general conditions that for $n$ large the distribution of $X$ may be approximated by a Poisson distribution, thus confirming Knox's conjecture. These conditions we give elsewhere (Barton and David, 1966). The first two moments of $X$ can either be written down from our general formula or can be obtained simply by direct argument. Thus the first moment $\mathcal{E}(X)$ is

$$
\mathcal{E}(X)=\frac{N_{1 S} N_{1 T}}{N}=\frac{2 N_{1 S} N_{1 T}}{n(n-1)}
$$

and if $N_{2 T}$ denotes the number of times two timeadjacencies are contiguous* (e.g. 345 or 456 , or 357 , etc., in the diagram) with a similar definition for $N_{2 S}$, the second moment $\mathcal{E}\left(X^{2}\right)$ is

$$
\begin{aligned}
\mathcal{E}\left(X^{2}\right)=\frac{2 N_{1} N_{1 T}}{n^{(\mathrm{s})}}+\frac{4 N_{2 S} N_{2 T}}{n^{(3)}}+ \\
\frac{4}{n^{(4)}}\left[N_{1 S^{(2)}}-2 N_{2 S}\right]\left[N_{1 T^{(2)}}-2 N_{2 T}\right]
\end{aligned}
$$

where

$$
n^{(w)}=n(n-1)(n-2) \ldots(n-w+1) .
$$

- The technical term for this is adjacent. We have tried here to avoid the technical terminology of graph theory except where we explain it. An excellent text for non-mathematicians is Graphs and Their Uses by O. Ore, Random House, N.Y. (for School Mathematics Study Group).
The computing of the quantities $N_{1 S}, N_{1 T}, N_{2 S}, N_{2 T}$ can be reduced to simple rote. Consider either graph and let $\rho_{i}$ stand for the number of lines radiating from the $i^{\text {th }}$ point $(i=1,2, \ldots, n)$. Then

$$
N_{1}=\frac{1}{2} \sum_{i=1}^{n} \rho_{i}
$$

and

$$
N_{2}=\sum_{i=1}^{n} \rho_{i} C_{2}=\frac{1}{2} \sum_{i=1}^{n} \rho^{2}{ }_{i}-N_{1}
$$

From the complete data on Knox's 96 cases (i.e. with $n=96$ ), computation of these quantities and subsequently of the mean and variance-say $\sigma^{2}{ }_{X}$ gives

$$
\mathcal{E}(X)=5 / 6=0.833 ; \sigma_{X}^{2}=0.802,
$$

suggesting, since asymptotically the distribution of $X$ is Poisson, the Poisson approximation for $X$ is undoubtedly adequate.

Further confirmation of the adequacy of the Poisson approximation in this case is provided by a sampling experiment conducted by Dr M. C. Pike of the MRC Statistical Unit, who randomly allocated the 96 time-coordinates of the 96 cases to the 96 space-coordinates and counted the numbers of pairs which were adjacent both in time and space. He repeated this 2,000 times. The frequenciest with which different values of $X$ were observed are given in Table III, together with the corresponding frequency to be expected when 2,000 samples are drawn from a

\begin{tabular}{|c|c|c|c|c|c|c|c|c|}
\hline $\boldsymbol{X}$ & 0 & 1 & 2 & 3 & 4 & 5 & $6+$ & Total \\
\hline $\begin{array}{l}\text { Observed Freq. } \\
\text { Poisson Freq. }\end{array}$ & $\begin{array}{l}885 \\
869 \cdot 2\end{array}$ & $\begin{array}{l}722 \\
724 \cdot 3\end{array}$ & $\begin{array}{l}294 \\
301 \cdot 8\end{array}$ & $\begin{array}{l}81 \\
83 \cdot 8\end{array}$ & $\begin{array}{l}14 \\
17 \cdot 5\end{array}$ & $\begin{array}{l}3 \\
2 \cdot 9\end{array}$ & $\begin{array}{l}1 \\
0.4\end{array}$ & $\begin{array}{l}2,000 \\
2,000\end{array}$ \\
\hline
\end{tabular}
Poisson distribution with a mean of $5 / 6$.

TABLE III

COMPARISON OF ACTUAL FREOUENCY IN A SAMPLE OF 2.000 WITH EXPECTED POISSON FREQUENCY WITH MEAN 5/6; RANDOM ALLOCATION OF SET OF TIME CO-ORDINATES

\section{ANOTHER Test FOR INTERACTION}

Knox has remarked that the foregoing test for interaction is not entirely satisfactory in that information is lost by the dichotomies into adjacent and not-adjacent and he has proposed other tests in which he makes a more discriminating categorization. We propose here an adaptation of a test which we have already put forward in another context! (Barton and David, 1962a, b). As before, consider the time observations arranged consecutively.

\footnotetext{
+ We are grateful to Dr Pike for permission to publish this Table.

‡ Our thanks are due to Dr C. C. Spicer of the General Register Office who suggested we might adapt our test to the present situation.
} 
Let the total length of the period over which the observations are made be $l$, and let there be $n$ observations. Then the average length, $d$, of an interval will be $l /(n-1)$ when recording starts at the first observation and finishes with the last. [If the observations are taken over a fixed period (e.g. five calendar years) rather than between first and last cases, we take the average interval $d$ as $l /(n+1)$. This makes no essential difference-so long as the choice is made by a predetermined rule the procedure outlined below remains valid irrespective of the particular rule.] We will say that two consecutive observations form a cluster of two if the time-distance between them is less than $d$, three consecutive observations will form a cluster of three if the time distances between the first and second and between the second and third are each less than $d$, and so on. For example, in Knox's data with 96 cases, the total time period is 3,557 days, so that the average interval is $37 \cdot 44$ days. We define, accordingly, points closer together than $\mathbf{3 8}$ days as clustering; for example, the first ten observations given in $\S 2$ will be defined as forming clusters of 2,6 , and 2 cases:

$$
(1,2)-(3,4,5,6,7,8)-(9,10)
$$

The space-coordinates of individuals are now divided in groups according to the time clustering, and the whole set of $n$ space-coordinates is considered as a randomization set. If $\left(x_{i}, y_{i}\right)$ denotes the coordinates of the $i^{\text {th }}$ point in space, $i=1,2, \ldots, n$, referred to some arbitrary convenient grid of coordinates, we write:

and

$$
\bar{x}=\frac{1}{n} \sum_{i=1}^{n} x_{i}, \quad \bar{y}=\frac{1}{n} \sum_{i=1}^{n} y_{i}
$$

$$
m_{a b}=\frac{1}{n} \sum_{i=1}^{n}\left(x_{i}-\bar{x}\right)^{a}\left(y_{i}-\bar{y}\right)^{b}, \quad a, b=0,1,2, \ldots
$$

Regarding the $n$ space-coordinates as a randomization set means that for this set, $\bar{x}, \bar{y}$, and the $m$ 's are constants. Let $\bar{x}_{t}$ and $\bar{y}_{t}$ be the means of the $x$ and the $y$ members of the space-coordinates of the observations lying in the $t^{\text {th }}$ time cluster, and for brevity write:

$$
k_{1 t}=\bar{x}_{t}-\bar{x}, k^{\prime}{ }^{\prime}=\bar{y}_{t}-\bar{y} .
$$

Then, if there are $r_{t}$ observations in this $t^{\text {th }}$ cluster, and there are $h$ time clusters in all

$$
\text { (with } n=\sum_{t=1}^{n} r_{t} \text { ) }
$$

the suggested criterion is:

$$
Q=\left(\frac{n-1}{n-h}\right)\left[1-\frac{\sum_{t=1}^{h} r_{t}\left(k^{2}{ }_{1 t}+k^{\prime 2}{ }_{1 t}\right)}{n\left(m_{20}+m_{02}\right)}\right]
$$

This is the ratio of the average squared distance between space-points within clusters to the overall average. It will be small if there is any positive time and space interaction-where by positive interaction is meant a tendency of cases close in space to be also close in time-and large if there is negative interaction. If there is no interaction the distribution of $Q$ is obtainable, in principle, from the particular set of space-coordinates and the particular set of times of occurrence concerned. The assumption of no interaction is equivalent to the randomization assumption, which says that all possible allocations of the $n$ given times to the $n$ given space-points are equally likely. There are $n$ ! such allocations (where $n !=n \times(n-1) \times(n-2) \times \ldots \times 3 \times 2 \times 1)$, so that if $n$ is large it would be extremely laborious to calculate the distribution exactly. Fortunately there are good approximations to the distribution which are accurate enough for all practical purposes ( $c f$. Barton and David, 1962b). These require the calculation of the mean and variance of $Q$ under randomization. We have that the expected value of $Q$ is always 1 and, writing $\sigma^{2}{ }_{0}$ for the variance of $Q$,

$$
\begin{aligned}
\sigma_{Q}^{2}=2(h-1)\left\{\frac{\beta(n-1)^{(2)}-\alpha(n-1)+1}{n(n-2)(n-3)(n-h)}\right\}+ \\
\frac{\Delta(n-1)\{(n+1) \alpha-(n-1)(2 \beta+1)\}}{(n-2)(n-3)(n-h)^{2}}
\end{aligned}
$$

where

$$
\begin{aligned}
& \alpha=\left(m_{40}+2 m_{22}+m_{04}\right) /\left(m_{20}+m_{02}\right)^{2}, \\
& \beta=\left(m^{2}+2 m^{2}{ }_{11}+m^{2}{ }_{08}\right) /\left(m_{20}+m_{08}\right)^{2}, \\
& \Delta=\sum_{t=1}^{h}\left(\frac{1}{r_{t}}-\frac{h}{n}\right) .
\end{aligned}
$$

When $h$ is quite large, $Q$ is nearly normal, so that $(Q-1) / \sigma_{Q}$ may be referred to normal tables (e.g. if it exceeds 1.96 in absolute value it is significant at the 5 per cent. level). When $h$ is small (e.g. when $h-1$ is less than $3 \sigma_{o} n$, as a working rule), a better approximation is to refer

$$
\frac{(n-1)-(n-h) Q}{(h-1) Q}
$$

to tables of the $\mathrm{F}$ distribution with degrees of freedom $(h-1) \theta,(n-h) \theta$, where

$$
\theta=\frac{2}{(n-1)}\left\{\frac{(n-1)}{(n-h) \sigma^{2} Q}-1\right\}
$$

When there is positive interaction, $Q$ will be significantly smaller than 1 and conversely.

\section{Discussion}

It should be remarked that, whilst we have replaced the adjacent/not-adjacent in space dichotomy by the actual distances, we have not avoided the necessity of arbitrarily dividing the time dimension. In the case of testing a specific disease or abnormality for time-space interaction, it may be possible to suggest 
a more informed division of the time scale. Thus, when a disease is suspected to have a given incubation period, say $d$, a time interval of slightly more than this period would be a better interval to use for dividing the cases into time-clusters. The theory given above would still apply with $d$ in place of $l /(n-1)$.

A second aspect of the test which is worthy of notice is that it shares with Knox's statistic the property of testing interaction whilst being substantially unaffected by irregularities of the separate time and space distributions. This is, of course, essential where notifications are incomplete or erratic, where there is seasonal variation, and where the cases are geographically clustered in towns and villages. The test likewise does not require knowledge of the population at risk, its age structure and so on. On the other hand, where such ancillary information is available, the test as it stands will not be able to make use of this information and so will not be fully efficient. This emphasizes the rather drastic simplicity of the test, which suggests that it will require a large number of cases to give a reasonable chance of detecting real interaction. Particularly as such interaction, in the nature of things, will not be very marked.

\section{Numerical COMParison}

The application of the $Q$ test to Knox's 96 cases of childhood leukaemia in Northumberland and Durham (spanning 3,557 days) gives a critical interval of 37.4 days. This divides the time into 35 clusters. With this clustering the measured value of $Q$ was 1.0430 with $\sigma_{o}=0.0950$. This is only slightly more than would be expected by chance-the normal equivalent being $(Q-1) / \sigma_{Q}=0.452$. It is far from significant, and, indeed, in this case $Q$ exceeds 1 , whereas $Q$ has to be significantly less than 1 to indicate epidemicity.

A closer look at the data reveals the cause of the discrepancy between this result and that given by Knox's $X$-statistic to be that the latter sharply accentuates the effect of the two or three cases which were very close in both time and space. These cases do not dominate the $Q$-statistic to such a degree as they do the $X$-statistic.

A contrasting application is provided by cases of an undeniably epidemic disease, namely measles. We have taken the data of measles at the two Southall schools "A" and "B" given by MRC Special Report No. 283 (1954), (see the map on p. 3 and figures in Table 36 therein). Here there are 104 cases at each school over a period of 1,034 days, so that the critical interval is 4 days (i.e. 5 or more days separate each cluster). This yields eighteen time-groups, so that $h=18, n=208$. The $Q$-statistic takes the value 0.21758 and $\sigma_{Q}=0.01849$, so that $Q$ is 42.32 standard deviations below its mean. This is overwhelmingly significant: there is no need here for more refined methods such as the $F$ approximation discussed in $\$ 4$.

With cases 4 days apart or less classified as adjacent in time and cases at the same school classified as adjacent in space, we find:

$$
\begin{array}{ll}
N_{1 S}=104\left({ }^{(2)}=10712,\right. & N_{2 S}=1044^{(3)}=1,092,624 \\
N_{1 T}=1843, & N_{2 T}=40876
\end{array}
$$

with $X=1592$ cases adjacent both in time and space. From the formulae, $\mathcal{E}(X)=917 \cdot 05, \sigma^{2}{ }_{X}=400 \cdot 76$, so that $X$ is 33.72 standard deviations above its mean. Here it will be noticed that $\mathcal{E}(X)$ is quite different from $\sigma_{X}^{2}$, so that the Poisson distribution is not appropriate for Knox's statistic.

6.1. When we take 10 days as the distance separating the time groups-this being roughly the incubation period of measles and thus more suitable for separating epidemics-we find $Q=0 \cdot 32003$, $\sigma_{Q}=0.01125$ (with $h=10$ ) so that $Q$ is 60.45 standard deviations below the mean. This is even more overwhelmingly significant. In a similar manner, counting cases which are 9 days apart or less as being adjacent in time, we find:

$X=3076, N_{1 T}=3446, N_{2 T}=139,593$ (with $N_{1 S}$ and $N_{2 S}$ as previously) so that $\mathcal{E}(X)=1714.68$ and $\sigma^{2}{ }_{X}=659 \cdot 91$.

Here $X$ is 52.99 standard deviations above the mean.

6.2. It should be remarked, in the context of the foregoing calculations, that it is by no means necessary to have the use of an electronic computer in order to compute Knox's statistic. Indeed the calculationseven for the variance of $X$-are much more quickly achieved by hand.

6.3. It is also worthy of notice that, in situations such as gave rise to the data just treated, where there are two space locations only, the individuals in each time group are dichotomized into two space-location classes. Thus we may test for no interaction by the usual chi squared statistic-let us call it $\phi^{2}$-for the hypothesis of independence in a $2 \times h$ contingency table. In fact the tests $Q$ and $\phi^{2}$ are equivalent in this situation: being mathematically related by

$$
Q=\left(\frac{n-1}{n-h}\right)\left(1-\phi^{2} / n\right)
$$

On the other hand, it should be noted that with the particular time division adopted above many groups will be small-for example seven of the eighteen groups (when the critical interval is $\mathbf{5}$ days) have just 
one member. Thus here we cannot validly use the tables of percentage points of $\chi^{2}$ of 17 degrees of freedom to assess the significance of $\phi^{2}$. [In this case, $h-1=17$.]

For, although $\mathcal{E}\left(\phi^{2}\right)=17 \cdot 08$, which is near enough to the expected value of $\chi^{2}$ of 17 degrees of freedom, the variance of $\phi^{2}$ is $12 \cdot 47$, which is much less than 34 (the variance of $\chi^{2}$ of 17 degrees of freedom).

An additional peculiarity of the case with just two space locations is that distances have only two possible values which are put into a standard form. This is to say physical distances do not enter into the expression for $Q$. The two standardized distances are the same thing as an adjacent/not adjacent classification. Thus in this case the comparison between $X$ and $Q$ rests only on the structure of the time-grouping.

\section{REFERENCES}

Barton, D. E., and David, F. N. (1962a). Bull. Inst. int. Statist., 39, 158; 455. (33 $3^{\mathrm{e}}$ Session, Paris, 1961.) - (1962b). Ann. hum. Genet., 25, 323.

- (1966). "Research Papers in Statistics", pp. 445-459. Wiley, New York.

Hill, A. Bradford (1961). "Principles of Medical Statistics", 7th ed., p. 172. Lancet, London.

Knox, G. (1963). Brit. J. prev. soc. Med., 17, 121.

- (1964). Ibid., $18,17$.

Medical Research Council (1954). "Air Disinfection with Ultra-violet Radiation; Its Effect on Illness among School-children", by the Air Hygiene Committee. Spec. Rep. Ser. No. 283.

\section{CORRECTION}

It is regretted that in the article by J. WAKEFIELD and L. BARIC which appeared in the October issue (Brit. J. prev. soc. Med., 1965, 19, 151), the key to Fig. 1 (p. 153) was accidentally transposed. The correct version is shown below.

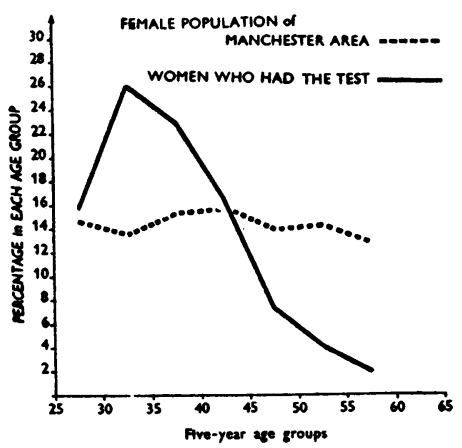

